\title{
Bernard Merdrignac, Hervé Bihan et Gildas Buron (dir.), À travers les îles celtiques
}

Per insulas scotticas. Mélanges en mémoire de Gwénaël Le Duc

\section{Magali Coumert}

\section{(2) OpenEdition}

\section{Journals}

Édition électronique

URL : http://journals.openedition.org/abpo/1856

DOI : $10.4000 /$ abpo. 1856

ISBN : 978-2-7535-1520-8

ISSN : 2108-6443

\section{Éditeur}

Presses universitaires de Rennes

\section{Édition imprimée}

Date de publication : 15 décembre 2010

Pagination : 198-199

ISBN : 978-2-7535-1309-9

ISSN : 0399-0826

\section{Référence électronique}

Magali Coumert, « Bernard Merdrignac, Hervé Bihan et Gildas Buron (dir.), À travers les îles celtiques », Annales de Bretagne et des Pays de l'Ouest [En ligne], 117-4 | 2010, mis en ligne le 14 janvier 2011, consulté le 22 septembre 2020. URL : http://journals.openedition.org/abpo/1856 ; DOI : https:// doi.org/10.4000/abpo.1856 
RoBIN, Guillaume, L'architecture des signes. L'art pariétal des tombeaux néolithiques autour de la mer d'Irlande, Rennes, PUR, 2009, 364 p., $27 €$.

Cet ouvrage sur l'art pariétal néolithique est la publication de la thèse de doctorat de Guillaume ROBIN (cotutelle Université de Nantes/University College Dublin, co-direction S. Cassen/M. O'Sullivan) soutenue, en anglais s'il vous plaît, à Nantes en novembre 2008.

Loin de se restreindre à l'exercice périlleux et réducteur de l'interprétation et de la signification des représentations pariétales, l'auteur analyse les formes géométriques souvent abstraites comme un complexe système de représentations structurant l'espace des tombes.

Ce sont ainsi 634 dalles gravées provenant de 89 monuments édifiés autour de la mer d'Irlande au cours du IVe millénaires avant J.-C. dont les signes et l'agencement /déploiement dans l'espace de la tombe sont passés en revue. La lecture est passionnante, très détaillée et fournie notamment en ce qui concerne les travaux antérieurs et les contextes archéologiques. L'important travail de remise aux normes graphiques actuelles permet au lecteur de consulter l'ensemble des données de manière objective et attentive.

Si l'organisation de l'ouvrage se veut académique, elle n'en est pas moins efficace. Aux cadres historique et méthodologique succède très vite un vaste travail de recensement comme en témoigne la seconde partie correspondant au répertoire des figures simples et complexes, des formes élémentaires aux variantes. Loin d'être une simple recherche à partir de sources iconographiques connues, l'auteur a effectué plusieurs voyages d'études afin de compléter et/ou étoffer au mieux cette base documentaire, en partie renouvelée.

Cœur de la réflexion et du travail de recherche, la relation art pariétal et espace architectural participe à la modélisation de la représentation spatiale déterminant non seulement l'emplacement des signes mais également celui des structures du monument (zones de passage, de rupture) et des dépôts funéraires.

Les propositions sur la position théorique initiale de dalles en réemploi préalablement ornées, mais de fait désormais spatialement désorganisées puisqu'en position secondaire, trouvent leur parallèle avec les hypothèses soulevées par certaines architectures funéraires contemporaines continentales, notamment bretonnes.

Le délai de parution de cette thèse, tout juste un an après sa soutenance, est suffisamment rare pour être souligné. Cette sérieuse synthèse fournie par Guillaume ROBIN intéressera, n'en doutons pas, plus d'un lecteur!

Jean-Noël GUYODO

Université de Nantes - UMR 6566 CReAAH UFR d'Histoire, Histoire de l'Art et Archéologie

JANKULAK, Karen, Geoffrey of Monmouth, University of Wales Press Series - « Writers of Wales », Cardiff, 2010, 125 p.

Les cinq précédents volumes de la collection "Writers of Wales » publiés par I'University of Wales Press présentent au grand public britannique des figures marquantes de la littérature nationaliste galloise contemporaine, dont, pour la plupart, les noms (de James Kitchener Davies à Herbert Williams) ne sont guère connus des lecteurs français. Le livre du Dr Karen Jankulak [KJ, par la suite], Lecturer à I'University of Wales - Trinity, Saint David (Lampeter) et Director du MA of Arthurian 
Studies, consacré à Geoffroy de Monmouth (Galfridus Monemutensis, † 1155) trouve cependant toute sa place dans cette collection. En effet, les œuvres de cet " historien » de la première moitié du XII ${ }^{\mathrm{e}}$ siècle (Historia Regum Britanniae, Prophetiae Merlini, et, dans une moindre mesure, Vita Merlini) ne relèvent pas seulement de I'histoire littéraire médiévale; elles ont influencé en profondeur des pans entiers de la littérature européenne jusqu'à nos jours. On peut donc souscrire - de loin, avec sympathie - à la remarque de $\mathrm{KJ}$ en fin de son introduction, comme quoi, une quinzaine d'années après la parution de la brillante synthèse de Michael J. Curley, Geoffrey of Monmouth, dans la «Twayne's English Authors Series » (New York, 1994), un retour de balancier replaçant Geoffroy au nombre des « écrivains du Pays de Galles » n'a rien d'inopportun aujourd'hui.

Très dense et de lecture agréable, ce petit livre (125 p.) est un modèle de vulgarisation intelligente. À ce titre, il vaut déjà de retenir l'attention des lecteurs des ABPO. Les normes de la collection excluent le recours aux notes infra-paginales conformes aux usages académiques. Toutefois, il n'y a qu'à se reporter à l'Index et à la "Select Bibliography » (présentée commodément chapitre par chapitre) pour être assuré de la qualité impeccable des informations mobilisées par l'auteur. Voici une mise au point parfaitement à jour des travaux récents des chercheurs britanniques sur le sujet.

Sans doute d'origine galloise ou bretonne (?), Geoffroy de Monmouth dont sont retracées ici les grandes lignes de la carrière comme magister et chanoine séculier d'Oxford, figure, en tant qu'évêque de Saint Asaph [PdG] (où le contexte politique ne lui a probablement jamais permis de se rendre) parmi les témoins du traité de Westminster entre Henri II et Etienne de Blois (1153) mettant un terme à la guerre civile qui avait opposé, depuis 1138, ce dernier à l'impératrice Mathilde, mère du Plantagenêt. Avec ses alliés gallois, le puissant comte Robert de Gloucester, protecteur de Geoffroy à qui celui-ci dédie l'HRB, s'est rallié au parti de la fille d'Henri ler Beauclerc. L'accent mis dans l'HRB sur les aspirations à la concorde entre Bretons est donc d'une brûlante actualité au moment de la composition de l'ouvrage (1139).

À l'encontre des préjugés négatifs qui dominent chez ses contemporains comme Guillaume de Malmesbury († v. 1143) et Henri de Huntingdon († 1160), Geoffroy de Monmouth s'assigne pour objectif de magnifier le passé de la Bretagne. La connaissance approfondie par KJ de la production pseudo-historique et prophétique galloise lui permet d'analyser subtilement les procédés narratifs mis en œuvre par Geoffroy pour recycler ses sources. Le présent CR ne saurait avoir la prétention de reprendre les développements serrés de l'auteur. Les chapitres 2 (" Historia Regum Britanniae and its sources") et 3 ("Geoffrey's models : Thinking about History in Medieval Wales and Ireland ») ont l'insigne mérite de sortir du cercle vicieux de l'interminable débat sur la part d'historicité ou d'invention de I'HRB en relativisant les termes de celui-ci.

Contentons-nous ici de pointer quelques éléments saillants de l'argumentation. Le fameux "livre très ancien en langue bretonne » (britannici sermonis : " gallois », « cornique » ou " breton armoricain »?) dont la réalité reste contestée et que l'archidiacre Gauthier d'Oxford aurait communiqué à Geoffroy est surtout invoqué par ce dernier comme argument d'autorité, sans doute indispensable pour contrebalancer le poids de l'Histoire Ecclésiastique de Bède le Vénérable. En réinterprétant le De Excidio de Gildas et l'Historia Britonnum attribuée à Nennius (v. 830) qu'il combine avec les apports de l'« histoire de synthèse " pratiquée en pays celtiques (que l'on pourrait schématiquement définir comme le calibrage, à partir de synchronismes artificiels, des histoires locales sur l'histoire universelle revue selon les grilles de la Bible), Geoffroy parvient à élaborer un récit linéaire qui se veut historiquement plausible. La légende de Brutus et des origines troyennes des Bretons revient à pla- 
cer la Bretagne sur un plan d'égalité avec Rome; l'introduction de la civilisation dans l'île est donc bien antérieure à la conquête. De même, Maxime, proclamé empereur par ses troupes de 383 à 388, et devenu Maxen Wledic dans la tradition historicolégendaire ultérieure fonde (paradoxalement, somme toute, pour un usurpateur) la légitimité des petits royaumes gallois sur l'héritage de l'Empire chrétien.

La transition entre passé romain et Bretagne post-romaine est incarnée par la figure d'Arthur, démarquée de I'Historia Britonnum. En inscrivant chronologiquement l'action de ce personnage dans la succession de personnalités historiques incontestables (tels que Carausius et Maxime), Geoffroy donne le coup d'envoi à une carrière littéraire pluriséculaire. Par contre, la croyance au retour salvateur d'Arthur pour mettre un terme aux déboires des Bretons, pourtant amplement attestée par des témoignages contemporains, ne trouve pas place dans I'HRB, ce qui a d'ailleurs permis aux Plantagenêts de la récupérer ultérieurement à leur profit, à partir de la découverte de sa tombe (1191) à Glastonbury. Ce sont ici Cadwaladr et Cynan (alias Conan Mériadec), déjà associés auparavant dans la littérature poétique galloise, qui sont porteurs de ces aspirations.

Le chapitre 6 («Magnus Maximus and the Colonization of Brittany ») dans lequel $\mathrm{KJ}$ explore les tenants et aboutissants de la légende de Conan constituerait, si cela était encore nécessaire, une raison supplémentaire de recommander ce livre aux lecteurs des ABPO. Ce chapitre fait en effet fort utilement bénéficier le public anglophone des apports de la recherche régionale au cours des trois dernières décennies, à la suite notamment de la publication des Origines de la Bretagne par Léon Fleuriot (1980). Relevons, au passage, la savoureuse expression "Breizo-latin » forgée par KJ pour qualifier les textes hagiographiques qui, comme les Vitae de saint Gurthiern et de saint Goueznou, ont alimenté les discussions sur l'existence éventuelle de traditions pré-galfridiennes en Bretagne. Voici un élégant néologisme, garant de la familiarité de l'auteur avec ce dossier complexe! Sa contribution (" la matière de Bretagne que Geoffroy de Monmouth n'a pas utilisée : les mythes d'origine des royaumes gallois médiévaux ») à l'ouvrage collectif dirigé par Magali Coumert et Hélène Tétrel, Histoires des Bretagnes - 1. Les mythes fondateurs, Brest, CRBC, 2010, apporterait une illustration récente de la collaboration engagée depuis des années par $\mathrm{KJ}$ avec les spécialistes de la question dans les centres de recherche de la région. C'est pourquoi, il serait injuste de part du signataire du présent $C R$ de tenir rigueur à l'auteur de n'avoir retenu dans la bibliographie correspondante aucun titre des publications françaises dont ce chapitre est à juste titre tributaire. II est permis toutefois de regretter que des contraintes éditoriales (aisées à comprendre) ne lui aient pas laissé la possibilité de faire état de ces échanges fructueux, d'autant que Karen Jankulak ne manque pas, avec délicatesse, de rendre hommage dans son avant-propos à la mémoire du professeur Gwénaël Le Duc à qui ce livre est amicalement dédié.

Bernard MERDRIGNAC

Merdrignac, B., Bihan, H., Buron, G., (dir.), À travers les îles celtiques. A-dreuz an inizi keltiek. Per insulas scotticas. Mélanges en mémoire de Gwénaël Le Duc, Britannia monastica 12 - Klask, Rennes, 2008, 502 p.

Cet ouvrage est dédié à la mémoire du professeur Gwénaël Le Duc qui s'est éteint en 2006, à l'âge de 55 ans. Il rassemble les contributions des " collègues, disciples et amis » et tire son unité de la personnalité du défunt. Ses centres d'intérêt éclectiques sont détaillés par la bibliographie initiale, qui rappelle que Gwénaël Le 